\title{
Morphological Adaptation after Bilateral Lung Transplantation in a Patient with Kartagener Syndrome: A Case Report
}

\author{
Chang Hun Kim, M.D., Bong Soo Son, M.D., Joohyung Son, M.D., Do Hyung Kim, M.D. \\ Department of Thoracic and Cardiovascular Surgery, Pusan National University Yangsan Hospital, Medical Research Institute of Pusan National University, \\ Yangsan, Korea
}

\section{ARTICLE INFO}

Received July 29, 2021

Revised September 26, 2021

Accepted October 7, 2021

Corresponding author

Do Hyung Kim

Tel 82-55-360-2127

Fax 82-55-360-2157

E-mail yumccs@nate.com

ORCID

https://orcid.org/0000-0002-8774-3397

\begin{abstract}
Kartagener syndrome (KS) is a rare disease with an incidence of 1 in 20,000 to 30,000 births. There is no cure for KS, and conservative medical treatments are used to relieve symptoms and prevent disease progression. Lung transplantation (LT) is the only treatment option for end-stage KS. Since patients with KS have anatomical abnormalities such as situs inversus totalis, which often require surgery to correct, most reports are related to surgical techniques. Reports about morphological adaptations and changes in transplanted lung structure after LT in patients with KS are rare. We performed LT in a patient with KS and observed morphological adaptation of the lungs for 6 months on chest computed tomography using a quantitative evaluation tool (Chest Image Platform; Harvard University Disability Resources, Cambridge, MA, USA).
\end{abstract}

Keywords: Kartagener syndrome, Situs inversus totalis, Lung transplantation, Case report

\section{Case report}

A 54-year-old man with Kartagener syndrome (KS), requiring long-term supplemental oxygen therapy and macrolide therapy due to recurrent lung infections for more than 10 years, was considered for bilateral lung transplantation (LT). Computed tomography (CT) of the chest showed bronchiectasis and pulmonary cystic changes pre- dominantly in the right lung, as well as situs inversus totalis (Fig. 1A). The patient underwent bilateral LT, with the donor being a 37-year-old woman who had died of nontraumatic cerebral hemorrhage. The donor had an arterial oxygen partial pressure/fraction of inspired oxygen ratio of 380. The estimated vital capacity-based size match of the donor lungs to the recipient lungs was $94 \%$. The operation was performed through a bilateral anterolateral thoracoto-
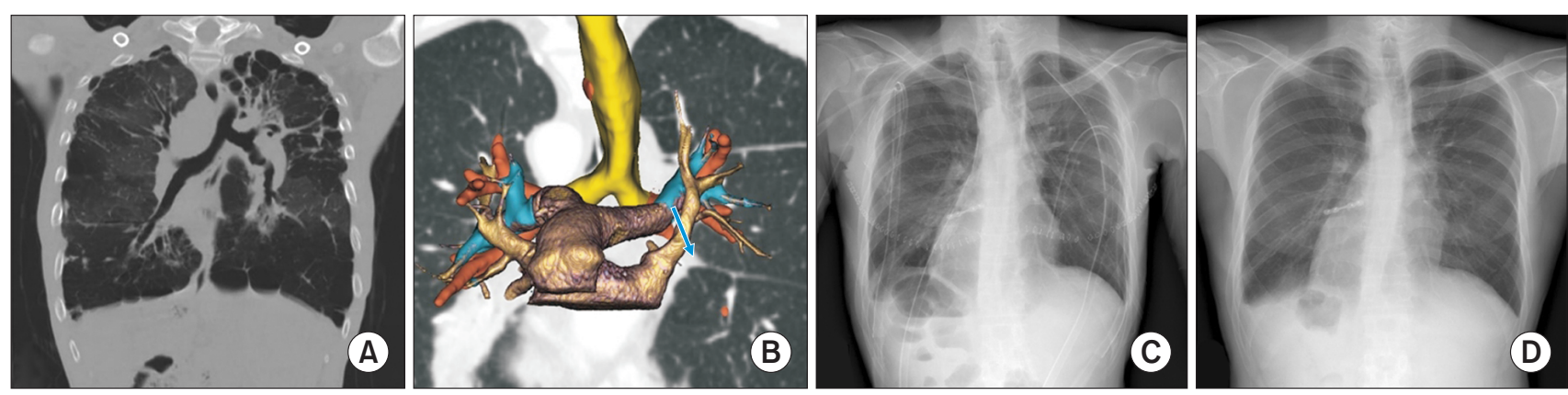

Fig. 1. (A) Preoperative chest computed tomography (CT) shows bronchiectasis and pulmonary cystic changes predominantly in the right lung, as well as situs inversus totalis. (B) A 3-dimensional-reconstructed postoperative chest CT scan shows the left pulmonary artery pathway changing abruptly from up to down at the level of the main bronchus (blue arrow and blue-colored structure). (C) A chest X-ray examination performed 1 week after surgery. (D) A chest X-ray examination performed 6 months after surgery. At both time points, there was no definite elevation of the diaphragm, suggesting the absence of phrenic nerve palsy. 
my. Severe pleural adhesions, especially at both apices, necessitated careful adhesiolysis before establishing extracorporeal membrane oxygenation (ECMO). Dual-drain peripheral ECMO with a pulmonary artery drain was performed to obtain complete drainage during transplantation. After bilateral pneumonectomy, the donor's left lung was first transplanted. The recipient's left pulmonary artery (LPA) was located anterior to the left main bronchus, while the donor's LPA was located superior to the donor's bronchus. We applied the method proposed by the Washington group for LPA anastomosis in the patient with KS [1]. With adequate mobilization of both the donor's and recipient's LPAs, the ends of both LPAs were beveled to match the size and orientation. For the transplantation of the right lung, the right pulmonary artery (RPA; anatomically LPA) of the recipient located superior to the right main bronchus was anastomosed as proximally as possible to reduce anatomical mismatch (Fig. 1B). The patient had an uneventful postoperative course and was discharged from the hospital on postoperative day 25. Chest X-ray examinations performed 1 week and 6 months after surgery did not show elevation of the diaphragm, which would have suggested phrenic nerve palsy (Fig. 1C, D).

Chest CT was performed 1 week and 6 months after sur- gery. Three-dimensional (3D) reconstruction was performed using 3D slicer software (http://www.slicer.org) to observe any deformation of the transplanted lungs and blood vessels. Additionally, the Chest Image Platform (CIP, a 3D slicer extension program; Harvard University Disability Resources, Cambridge, MA, USA) was used for a quantitative evaluation of the volume and mass of the lung. A CT scan performed 1 week after surgery showed a severe stricture due to kinking of the pulmonary artery by an abnormal pathway and extrinsic compression of the left main bronchus (Fig. 2A, B). However, a CT scan performed 6 months postoperatively showed an increase in the size of the narrowed LPA (Fig. 2C, D). We found that the vascular stricture gradually resolved with time. Additionally, on 1-week postoperative CT, the right lower lobe (RLL) accounted for $27.7 \%$ and $35.45 \%$ of the total lung volume and mass, respectively, and the corresponding proportions in the right middle lobe (RML) were 29.79\% and 27.04\% (Fig. $3 \mathrm{C}, \mathrm{D})$. Based on these assessments of lung volume and mass, we interpret that the right deviation of the heart due to situs inversus totalis led to collapse of the RLL and subsequent overexpansion of the RML in the early postoperative period. The mass ratio on chest CT was similar at 6 months, but the ratio of volume increased from $27.70 \%$ to
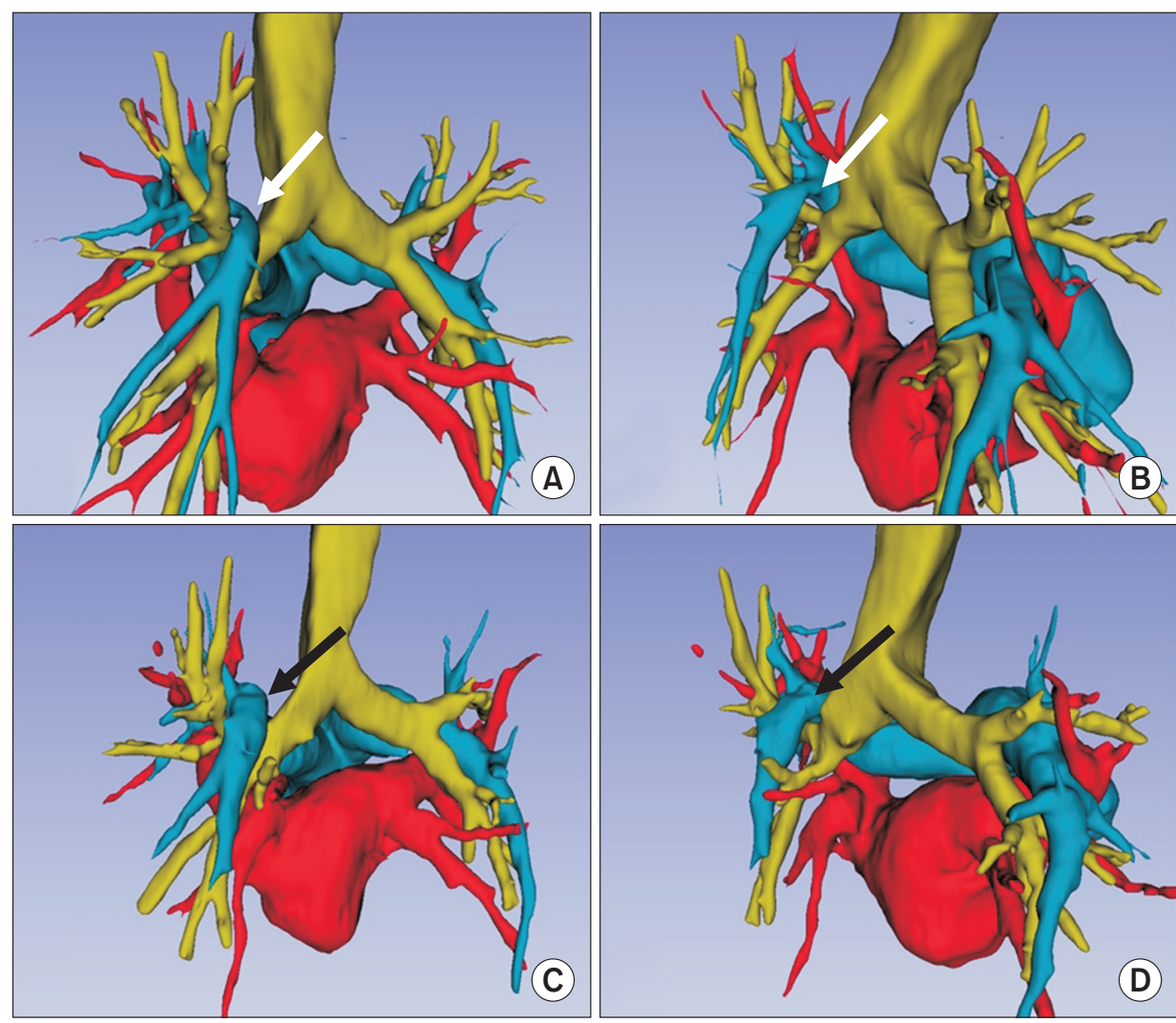

Fig. 2. Chest computed tomography (CT) scans. (A, B) A CT scan taken 1 week after surgery shows a severe stricture (white arrow) due to the kinking of the pulmonary artery by an abnormal path and extrinsic compression of the left main bronchus. (C, D) A CT scan taken 6 months postoperatively shows an increase in the size of the narrowed pulmonary artery (black arrow). 

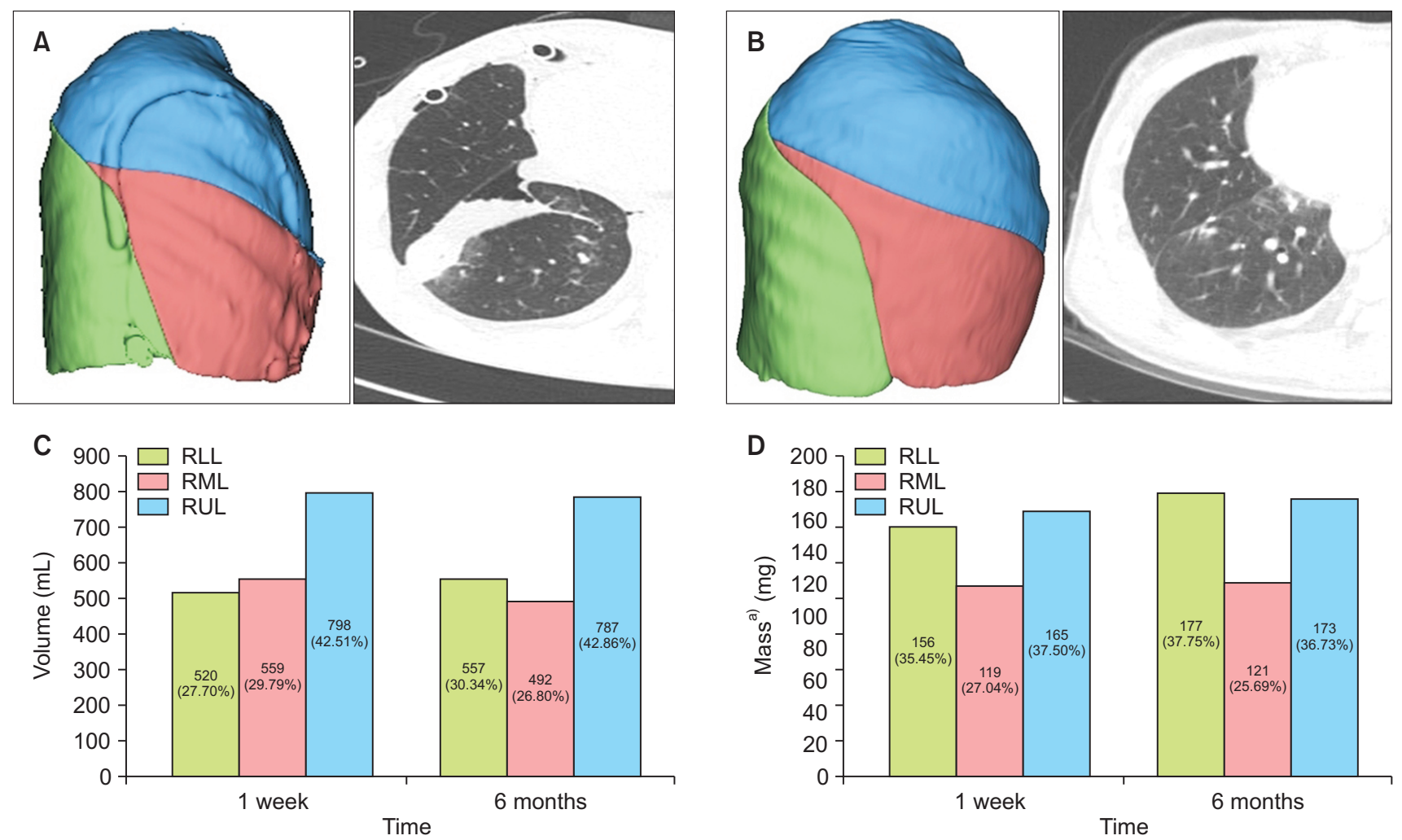

Fig. 3. The 3-dimensional-reconstructed computed tomography (CT) scans at 1 week and 6 months postoperatively show that morphological adaptation occurred gradually, but the lung size in the right lower lobe (RLL) did not fully recover to its normal size. (A) The CT scan at 1 week showed that the RLL (green area) was small compared to the upper and middle lobes, and focal atelectasis was seen on chest CT. (B) The CT scan at 6 months shows that the atelectasis had disappeared, but not enough to recover to normal size. (C) While the volume of the RLL was lower than that of the right middle lobe (RML) according to the chest CT examination performed at postoperative week 1, the volume of the RLL increased more than the volume of the RML at 6 months postoperatively. However, the volume of the RLL did not increase to its normal value (i.e., 40\%). (D) The mass of the RML and RLL was similar after 6 months. Thus, the RLL increased

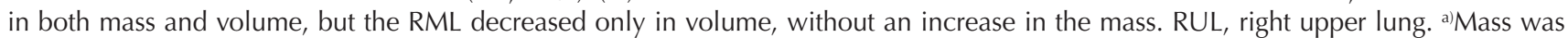
calculated from the following formula: voxel tissue weight=[1-(voxel density/-1,000)]×voxel volume.

$30.43 \%$ in the RLL and decreased from $29.70 \%$ to $26.80 \%$ in the RML. The atelectasis in the RLL disappeared with time and its volume increased, but not to the normal size. Additionally, the hyper-expanded RML showed a tendency to change to a normal size over time (Fig. 3A, B).

The patient involved in this case report authorized the disclosure of his protected health information for academic purposes.

The institutional review board at Pusan National University Yangsan Hospital approved the present study (IRB approval no., 05-2021-175), and the patient consent was waived for the retrospective data analysis.

\section{Discussion}

LT in patients with KS should be performed as if both lungs are to be transplanted contralaterally, because KS presents with dextrocardia, bronchial asymmetry, and anatomical variations of major vascular structures due to situs inversus totalis. Therefore, anatomical considerations due to situs inversus totalis are important factors in successful LT in patients with KS.

The first consideration is the pathway and mismatch of the pulmonary artery of the donor and recipient. In situs inversus totalis, the main pulmonary artery is divided in the anterior portion of the right main bronchus, and the LPA runs below the right aortic arch and along the anterior of the left main bronchus, whereas the RPA runs through the superior aspect of the right main bronchus and runs along the posterior aspect of the right main bronchus. Therefore, a mismatch of the pulmonary artery pathway between the donor and recipient is inevitable when a normal lung is transplanted in a patient with KS [2-4]. Especially on the left side, this anatomical mismatch causes 
fundamental structural problems after transplantation; the LPA runs upwards, turns around the left main bronchus, and runs downward towards the major fissure. The pulmonary artery pathway changes abruptly from upwards to downwards at the level of the main bronchus. The path of the pulmonary artery shows a reversed U-shape after transplantation, causing the LPA to kink.

The degree of kinking can be reduced by sufficiently mobilizing the donor and recipient pulmonary arteries to maintain a sufficient length of the pulmonary artery and by performing a bevel-type pulmonary artery anastomosis to allow the main bronchus to return as freely as possible [1]. However, this method is difficult. If kinking occurs despite all treatments, it is best to observe the patient's condition during follow-up rather than performing an additional operation to relieve vessel kinking. For this reason, we decided to closely observe this patient for vessel kinking. Chest CT performed 1 week after surgery showed an abrupt change in the direction of travel of the pulmonary artery and severe kinking in the left main bronchial region (Fig. 2A, B). However, a 6-month follow-up showed that the shape of blood vessels in the kinked part changed to become near-normal through gradual morphological adaptation (Fig. 2C, D).

Another consideration is the effect of morphological mismatch on the volume of the lung due to situs inversus totalis. Since the right-deviated heart in situs inversus totalis is located between the RML and RLL, the RML tends to over-expand and the RLL tends to shrink in the early postoperative period. According to a few reports on LT in KS patients, right lower lobectomy was performed to prevent volume-related atelectasis [5,6]. Recently, size-matched transplantation has been preferred over lobectomy because lobectomy increases the risk of a bronchial fistula [5]. Despite efforts to match size, atelectasis of the RLL may still occur due to an anatomical abnormality; in this case, the CT scan at 1 week showed atelectasis of the RLL (Fig. 3A). However, the CT scan at 6 months showed that atelectasis of the RLL had resolved as a result of the morphological adaptation of the RML and RLL (Fig. 3B). According to the data calculated from chest CT performed at postoperative week 1, the mass of the RML was less than that of the RLL (Fig. 3D), whereas the volume of the RML was higher than that of the RLL (Fig. 3C). However, on the chest CT scan taken 6 months postoperatively, the RLL had increased both in mass and volume, whereas the RML had decreased only in volume, and not in mass (Fig. 3C, D). The CT scans at 1 week and 6 months showed that the morphological ad- aptation gradually occurred, but the size of the RLL did not recover, considering that the normal volumes of the RML and RLL are around $25 \%$ and $40 \%$ of the right lung [6]. It is thought that situs inversus totalis continued to affect the volume of the RLL.

LT in patients with KS inevitably causes a morphological mismatch and structural abnormalities of the anastomotic vessels due to situs inversus totalis. Although it is thought that surgery is difficult or impossible due to anatomical incompatibility, based on our experience, if the characteristics of the anatomical structures and gradual morphological adaptation after surgery are understood, LT in patients with KS may be considered a viable option.

\section{Conflict of interest}

No potential conflict of interest relevant to this article was reported.

\section{ORCID}

Chang Hun Kim: https://orcid.org/0000-0003-3181-7454

Bong Soo Son: https://orcid.org/0000-0001-6681-6094

Joohyung Son: https://orcid.org/0000-0002-9144-5499

Do Hyung Kim: https://orcid.org/0000-0002-8774-3397

\section{References}

1. Gauthier JM, Takahashi T, Bierhals AJ, et al. Technical considerations for lung transplantation in Kartagener's syndrome. Ann Thorac Surg 2019;107:e337-9.

2. Macchiarini P, Chapelier A, Vouhe P, et al. Double lung transplantation in situs inversus with Kartagener's syndrome. Paris-Sud University Lung Transplant Group. J Thorac Cardiovasc Surg 1994;108:8691.

3. Wang B, Zhang X, Jiang W, et al. Double lung transplantation for end-stage Kartagener syndrome: a case report and literature review. J Thorac Dis 2020;12:1588-94.

4. Hayes D Jr, Reynolds SD, Tumin D. Outcomes of lung transplantation for primary ciliary dyskinesia and Kartagener syndrome. J Heart Lung Transplant 2016;35:1377-8.

5. Brioude G, D'journo XB, Reynaud-Gaubert M, Thomas PA. Bronchial fistula after lobar size reduction for bilateral lung transplantation in Kartagener's syndrome: a surgical challenge. Interact Cardiovasc Thorac Surg 2013;17:184-6.

6. Mueller C, Hansen G, Ballmann M, et al. Size reduction of donor organs in pediatric lung transplantation. Pediatr Transplant 2010;14: 364-8. 